\title{
Динамическая оценка эффективности инвестиционных проектов с учетом особенностей газовой отрасли
}

\author{
Протасов B.C. ${ }^{13}$
}

В статье предложена методика оценки эффективности инвестиционных проектов, модифицированная для использования на каждой стадии жизненного ичкла проекта. Ее использование позволит проводить оценку проектов в динамике, обеспечивая рекомендации по методическим улучшениям и практические советы при реализации новых проектов. Методика адаптирована для учета особенностей газовой отрасли.

$J E L: G 32, O 22$

Ключевые слова: оченка эффективности, проектный анализ, инвестищионный проект, $2 a 3$

\section{Введение}

Перед Россией в настоящее время стоит ряд задач, выполнение которых необходимо для обеспечения устойчивых темпов роста и развития: повышение производительности труда и капитала (ВЭФ, 2011), а также увеличение энергетической эффективности (Башмаков, 2011). Эти задачи можно объединить в одну - повышение эффективности. Ocобое значение при этом имеет повышение эффективности работы российской нефтегазовой отрасли, обеспечивающей более $20 \%$ ВВП и $60 \%$ экспорта страны (Башмаков, 2011). В будущем, в условиях стабилизации добычи нефти и роста добычи газа в России (Энергетическая стратегия - 2030, 2009), все больший вес в экономике России будет приобретать газовая составляющая. В российскую газовую отрасль в 2011-2030 годах может быть инвестировано порядка 510-630 млрд долларов ${ }^{14}$ (Энергетическая стратегия - 2030, 2009; International Energy Agency, 2010). Но российские газовые проекты подвергаются значительной критике за их низкую эффективность (Клейнер, 2006; Милов, 2007; Игнатьев, 2010; Larsson, 2007; Baev, Overland, 2010). Это обуславливает необходимость разработки методики оценки эффективности для газовых проектов с учетом их специфики, которая позволила бы не только провести детальную объективную оценку эффективности проектов, но и сравнить ее фактический уровень с проектными значениями, то есть рассмотреть процесс оценки в динамике.

Ряд исследователей указывает на необходимость проведения динамической оценки эффективности проектов на различных стадиях их реализации (Виленский, Лившиц, Смоляк, 2008; Anbari, 1985; Cano, 1992; Huseby, Skogen, 1992; Erikstad et. al., 1998; Belli et. al., 1998; Dey, 2002; Xie et.al., 2010). Произошедшие в последние годы резкие изменения мировой и российской экономической ситуации, увеличение волатильности ряда ключевых показателей, оказавшие значительное влияние на параметры инвестиционных проектов, только подтверждают это. Особое значение при проведении динамической оценки имеет сравнение плановых и фактических показателей эффективности, учитывая явление завышенных ожиданий: уровень коммерческой эффективности 1100 проектов Мирового банка через 5-8 лет в среднем оказывался на 7-10 процентных пунктов ниже, чем было заложено в проект (Kaufman, 1991). Систематическая переоценка эффективности и недооценка рисков проекта вызвана как поведенческими и политическими, так и методическими причинами (Лившиц, Швецов, 2011), что обуславливает возможность

\footnotetext{
${ }_{13}^{13}$ Аспирант экономического факультета НИУ ВШЭ.

${ }^{14}$ В ценах 2009 года.
} 
снижения уровня расхождений плановых оценок и фактических значений благодаря оптимизации методик оценки эффективности.

\section{Теоретические и методические основы динамической оценки эффективности}

Под динамической оценкой эффективности инвестиционного проекта в настоящем исследовании понимается проведение регулярной взаимоувязанной переоценки эффективности проекта на всех стадиях его жизненного цикла. Регулярная переоценка необходима для получения возможности реагировать на появляющиеся угрозы и обеспечения эффективного управления проектом. При этом каждая новая оценка должна сравниваться с предыдущими для получения практических и методических рекомендаций. Проведение оценки эффективности является трудоемким и длительным процессом, поэтому для ее оптимизации методика должна быть адаптирована к каждой стадии жизненного цикла проекта во избежание лишних затрат.

Наиболее подробные классификации жизненного цикла проекта могут включать в себя стадии генерации идеи (идентификации проекта), предварительной оценки, разработки проекта (подготовки технико-экономического обоснования), экспертизы и одобрения проекта, переговоров по нему, реализации (строительства), ввода в эксплуатацию, эксплуатации, оценки результатов проекта, ликвидации (Labuschagne, Brent, 2006; Андреев, 1997; Lester, 2007) и ряд других. Интегрируя различные варианты, можно выделить до девятнадцати последовательных стадий, но в большинстве исследований жизненный цикл проекта разделяется на более крупные интервалы: от пяти до восьми стадий. Следует отметить, что рядом авторов выделяются специфические классификации жизненных стадий проекта в зависимости от отрасли, в том числе нефтегазовой (Зубарева, 2001).

С методической точки зрения слишком подробное деление жизненного цикла проекта при проведении динамической оценки его эффективности не представляется оправданным, так как между соседними стадиями будут наблюдаться лишь незначительные методические отличия или их не будет вовсе. Для целей настоящего исследования предлагается разделить жизненный цикл проекта на три стадии:

1. Подготовки, которая определяется как стадия от выдвижения идеи проекта и до принятия окончательного инвестиционного решения (ОИР).

2. Реализации - от принятия ОИР до ввода проекта в эксплуатацию.

3. Эксплуатации - от ввода проекта в эксплуатацию до его ликвидации.

Следует отметить, что предложенное деление жизненного цикла проекта схоже, в частности, с общей классификацией Мирового банка (МБ) (Андреев, 1997) и классификацией оценки проектов Клиланда (Cleland, 1985). Для большинства крупных проектов о принятии ОИР объявляется публично, что облегчает задачу различения стадий для независимого исследователя. При необходимости на каждой из стадий оценка может производиться многократно, но методика проведения оценки внутри каждого этапа предполагается неизменной.

Значительное число исследователей и такие организации, как Мировой банк указывают на обязательность проведения завершающей оценки проекта (Anbari, 1985; Cleland, 1985; Андреев, 1997; Busby, 1999; Gunton, 2003; Anbari et.al., 2008), в то время как действующие российские Методические рекомендации (Методические рекомендации, 2000) этого не требуют. Завершающая оценка должна показывать эффективность выполненного проекта, объяснять причины основных отклонения фактических показателей от плановых и выдавать рекомендации для увеличения эффективности реализации последующих проектов (Anbari, 1985; Cleland, 1985).

Возможные различия между методиками оценки эффективности инвестиционного проекта систематизированы в таблице 1. Ключевое методическое различие между проведением оценки эффективности на различных стадиях заключается в ее основной цели. На первой стадии должна проводиться оценка целесообразности инвестиций в один из 
альтернативных вариантов проекта. С каждым годом после принятия окончательного инвестиционного решения объем будущих инвестиций снижается, а приведенная к дате оценки стоимость будущих денежных притоков увеличивается. Поэтому, если проводится оценка не венчурных проектов, на второй и третьей стадии оценки закрытие проекта возможно только при радикальных изменениях внутренней или внешней среды (или при допущении грубой ошибки на первой стадии). Мониторинг подобных радикальных изменений, а также соответствия предложенных на первом этапе предпосылок фактическим значениям показателей представляется важным на втором этапе (Виленский, Лившиц, Смоляк, 2008). В то время как на этапе эксплуатации в первую очередь необходимо получить завершающую (апостериорную, фактическую) оценку проекта, то есть определить итоговую эффективность его работы с начала жизни проекта до момента оценки, с тем чтобы определить перспективность реализации аналогичных проектов в будущем и корректность использованных методик и предпосылок. Многие исследователи указывают на необходимость проведения оценки фактической эффективности по завершении проекта (Anbari, 1985; Виленский, Лившиц, Смоляк, 2008; Волков, Грачева, 2009). При этом следует учитывать, что завершением проекта для некоторых его участников может быть не только его ликвидация, но и ввод проекта в эксплуатацию, завершение заемного финансирования проекта или его выход на проектную мощность. Кроме того, у целого ряда проектов, в том числе газопроводных, срок жизни может превышать 30-50 лет, поэтому проведение апостериорной оценки по их фактическому завершению (ликвидации) будет иметь скорее историческую, чем практическую ценность. В то же время ряд авторов (Зубарева, 2001; Андреев и др., 2002) рекомендуют проведение завершающей оценки проекта не менее чем через 5-7 лет после начала его эксплуатации (при сроке жизни проекта в 50 лет).

Поэтому с практической (выводы по аналогичным проекта на более ранней стадии) и теоретической (модификация существующих методик) точек зрения представляется оправданным проводить апостериорную оценку еще на стадии эксплуатации. Следует учитывать, что от выбора точки проведения завершающей оценки может зависеть ее результат (Olsson et.al., 2010), поэтому она должна определяться индивидуально для каждого проекта.

Таблица 1

Сравнительная характеристика проведения оценки на различных стадиях проекта

\begin{tabular}{|l|l|l|l|}
\hline Характеристика / Стадии & $\mathbf{1}$ - Подготовка & 2- Реализация & 3-Эксплуатация \\
\hline $\begin{array}{l}\text { Первоочередная цель } \\
\text { проведения оценки }\end{array}$ & $\begin{array}{l}\text { Оценка } \\
\text { целесообразности } \\
\text { инвестиций в } \\
\text { проект }\end{array}$ & $\begin{array}{l}\text { Мониторинг } \\
\text { состояния и } \\
\text { рисков проекта }\end{array}$ & $\begin{array}{l}\text { Проведение } \\
\text { завершающей } \\
\text { оценки }\end{array}$ \\
\hline Прошлые издержки & Не учитываются & Не учитываются & Учитываются \\
\hline Сравнения & Нет & $\begin{array}{l}\text { Сравнение План } \\
\text { План, План - } \\
\text { Факт }\end{array}$ & $\begin{array}{l}\text { Сравнение План - } \\
\text { Факт }\end{array}$ \\
\hline Степень неопределенности \\
\hline $\begin{array}{l}\text { Сложность методик и } \\
\text { факторово учитываемых }\end{array}$ & Максимальная & Средняя & Минимальная \\
\hline $\begin{array}{l}\text { Объем доступной } \\
\text { информации }\end{array}$ & Среднее & Максимальное \\
\hline $\begin{array}{l}\text { Детализация доходов и } \\
\text { расходов, продуктового } \\
\text { ассортимента }\end{array}$ & Минимальное \\
\hline $\begin{array}{l}\text { Экспертные оценки, данные } \\
\text { по аналогам }\end{array}$ & Максимально & Среднее & Максимальное \\
\hline
\end{tabular}




\begin{tabular}{|l|l|l|l|}
\hline $\begin{array}{l}\text { Оценка эффективности } \\
\text { проекта в целом }\end{array}$ & Да & Не обязательно & Да \\
\hline $\begin{array}{l}\text { Выбор среди } \\
\text { альтернативных вариантов }\end{array}$ & $\begin{array}{l}\text { Альтернативные } \\
\text { проекты и } \\
\text { маршруты }\end{array}$ & $\begin{array}{l}\text { Незначительные } \\
\text { отклонения от } \\
\text { основного } \\
\text { маршрута }\end{array}$ & Нет \\
\hline Относительные оценки & Да & Нет & Нет \\
\hline $\begin{array}{l}\text { Оценка технической } \\
\text { реализуемости }\end{array}$ & Да & Не обязательно & Не обязательно \\
\hline $\begin{array}{l}\text { Необходимость оценки } \\
\text { рисков }\end{array}$ & Да & Да & Не обязательно \\
\hline $\begin{array}{l}\text { Перечень рисков } \\
\text { учитываются все } \\
\text { риски }\end{array}$ & $\begin{array}{l}\text { Учитываются } \\
\text { все риски }\end{array}$ & $\begin{array}{l}\text { Не учитываются } \\
\text { риски, возникающие } \\
\text { на стадии } \\
\text { реализации проекта }\end{array}$ \\
\hline
\end{tabular}

Дифференциация целей оценки эффективности при проведении на различных этапах жизни проекта обуславливает ряд различий в методиках оценки на каждом этапе. В частности, денежные потоки прошлых периодов, в том числе необратимые издержки, которые не учитываются в стандартной методике оценки коммерческой эффективности (Виленский, Лившиц, Смоляк, 2008), должны приниматься во внимание при проведении завершающей оценки.

Два других ключевых отличия проведения оценки на различных стадиях жизни проекта: уровень неопределенности и объем доступной информации по проекту. При прочих равных наблюдается обратная зависимость степени неопределенности и прямая зависимость объема доступной информации от срока жизни проекта. Следует учитывать, что возможны исключения из правила для уровня неопределенности, обусловленные макроэкономическими циклами (если более поздняя стадия оценки приходится на период экономического кризиса, то уровень неопределенности может оказаться выше), периодом волатильности цен или политической нестабильностью. С методической точки зрения увеличение сложности и детальности используемых методик, а также числа факторов, принимаемых во внимание, должны обуславливать увеличение точности проводимой оценки (Методические рекомендации, 2000; Виленский, Лившиц, Смоляк, 2008). С другой стороны, в условиях высокой неопределенности и недостатка информации это может привести к увеличению ошибки. В условиях нехватки информации такие инструменты, как экспертные оценки и оценка по аналогичным проектам, должны использоваться часто, но по мере роста информации по проекту и окружающей его среде их доля среди всех используемых инструментов оценки должна снижаться.

На второй стадии в процессе мониторинга проведение общей оценки эффективности проекта (в отличие от эффективности для каждого участника) не является обязательной к проведению. И только если показатели эффективности участия предприятия оказываются меньше плановых показателей, рекомендуется провести оценку эффективности для всех участников проекта (Методические рекомендации, 2000). Однако это верно только в случае проведения оценки одним из участников проекта, в то время как при проведении оценки эффективности компанией-оператором проекта представляется обоснованным проведение оценки в целом и на второй стадии. Поэтому для стадии реализации алгоритм выглядит следующим образом (если оценку проводит третья сторона, то выбор остается на ее усмотрение): 


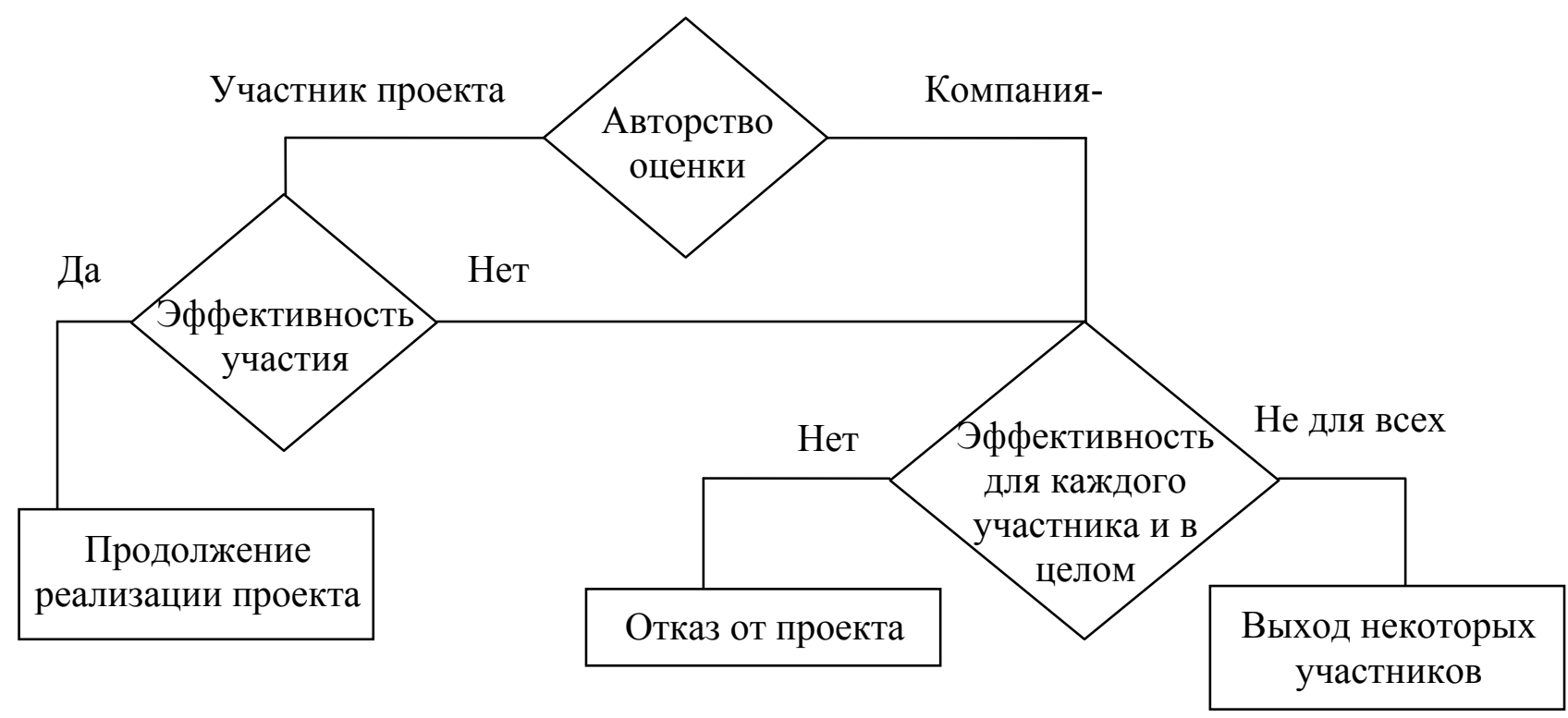

Рисунок 1. Алгоритм проведения оценки проекта в целом и для отдельных участников при проведении мониторинга (этап реализации)

На стадии эксплуатации снова необходимо проводить оценку проекта в целом для сравнения фактических и плановых показателей.

На первом этапе важной задачей оценки эффективности проекта является его сравнение с альтернативными проектами и вариантами маршрута (Belli et. al., 1998). После принятия ОИР необходимость в этом отпадает в силу невозможности выбрать альтернативный проект. На этапе реализации возможны лишь небольшие коррекции проекта при получении в ходе оценки данных о новых рисках ${ }^{15}$.

Оценка рисков наиболее важна на стадии подготовки проекта, затем ее значимость снижается (Belli et. al., 1998). На стадии реализации еще остаются возможности для корректировки проекта, но существенно меньшие, чем на стадии подготовки. Поэтому при выявлении рисков на поздних стадиях жизни проекта рекомендации по их избеганию или нивелированию последствий должны касаться в первую очередь воздействия на внешнюю среду, а не на внутреннюю. Если было принято решение о проведении оценки рисков на стадии эксплуатации, то в анализе не должны учитываться ряд специфических рисков, характерных для стадии реализации проекта. Они либо не исполнились, либо обусловили конкретный ущерб для компании, либо трансформировались в другие риски (например, риск негативного воздействия на популяцию промысловых рыб может трансформироваться в риск исков со стороны рыболовецких организаций). К числу рисков стадии реализации для подводных газопроводов можно отнести, в частности, риски увеличения стоимости проекта, неполучение разрешения на строительство, шумовой эмиссии при прокладке и.т.п.

\section{Специфика газовой отрасли и газопроводных проектов}

Магистральные газопроводные объекты обладают рядом особенностей, которые оказывают влияние на методики оценки эффективности и поэтому должны быть учтены при их разработке. Все особенности газопроводных проектов можно разделить на неотъемлемые и распространенные, а также характерные для нефтегазовой отрасли и трубопроводных проектов (таблица 2).

\footnotetext{
15 Например, маршрут газопровода «Северный поток» был изменен после ОИР (было принято решение об обходе острова Борнхольм с севера, а не с юга), когда выяснилось, что небольшой участок маршрута проходит по спорной акватории Германии и Польши, что могло привести к запрету или значительной задержке строительства. В результате протяженность газопровода выросла на 8 км, что увеличило расходы по проекту.
} 
Длительные сроки реализации газопроводных проектов ведут к тому, что внешние условия за это время могут поменяться сильнее, чем у менее продолжительных проектов. В результате для газопроводных проектов проведение динамической оценки является более актуальной задачей. При этом высокая капиталоемкость и длительные сроки реализации проектов ведут к высокой циклической зависимости отраслевых проектов (Лившиц, Швецов, 2011). Это обуславливает необходимость заложения в предпосылки значительных резервов на непредвиденные расходы, а также не просто проведение анализа чувствительности коммерческой эффективности к изменениям конъюнктуры, но построение полноценных альтернативных сценариев при проведении оценки.

Таблица 2

Матрица особенностей газопроводных проектов

\begin{tabular}{|c|c|c|}
\hline & Отраслевые & Трубопроводные \\
\hline Неотъемлемые & $\begin{array}{l}\text { - Высокая стоимость, } \\
\text { капиталоемкость } \\
\text { - Риски взрывов и утечек } \\
\text { - Значительные экологические } \\
\text { риски } \\
\text { - Высокая доля неуправляемых } \\
\text { факторов, преобладание } \\
\text { стохастических и } \\
\text { неопределенных факторов } \\
\end{array}$ & $\begin{array}{l}\text { - Географическая } \\
\text { детерминированность поставок } \\
\text { - Высокая доля основных фондов } \\
\text { - Низкая ликвидность активов } \\
\text { проекта } \\
\text { - Необратимость инвестиций }\end{array}$ \\
\hline Распространенные & $\begin{array}{l}\text { - Длительные сроки реализации } \\
\text { - Высокая потребность в заемных } \\
\text { средствах } \\
\text { - Высокая роль политических } \\
\text { факторов } \\
\text { - Многостадийность } \\
\text { - Ориентированность на экспорт }\end{array}$ & $\begin{array}{l}\text { - Большая протяженность } \\
\text { проектов } \\
\text { - Уникальность проектов (малое } \\
\text { число аналогов для сравнения) } \\
\text { - Труднодоступность для } \\
\text { технических проверок }\end{array}$ \\
\hline
\end{tabular}

Доля основных средств для проектов нефтегазового транспорта составляет около 97\% в среднем (Миловидов и др., 2006), что обуславливает возможность использования упрощенных методов для анализа движения оборотных средств при проведении оценки (Виленский, Лившиц, Смоляк, 2008). Высокая потребность в заемных средствах (Андреев и др., 2002) обуславливает необходимость анализа схемы финансирования проекта даже на ранних стадиях проекта.

Большая протяженность магистральных газопроводов означает наличие социальных и экологических последствия для многих регионов (соответственно, проведения оценки последствий проекта для каждого из них), а не для одного-двух в случае проекта разработки месторождения. Географическая детерминированность поставок по трубопроводу (в отличие от поставок танкерами, железной дорогой, автотранспортом) ограничивает возможности расширения рынков сбыта, что упрощает их анализ, но увеличивает рыночные и политические риски.

Труднодоступность для технических проверок ${ }^{16}$ (Dey, 2002) увеличивает технические риски, а также придает более высокую значимость проведению оценки технической реализуемости проекта. Высокие экологические риски обуславливают обязательность проведения оценки последствий для окружающей среды.

Для газовых и нефтяных проектов также распространенной является их реализация в несколько этапов. Это повышает актуальность проведения сравнений плановых и

\footnotetext{
${ }^{16}$ Так как трубопроводы большую часть маршрута могут быть расположены под землей или водой.
} 
фактических показателей. По итогам строительства первой нитки газопровода могут быть введены поправочные коэффициента и проведена переоценка эффективности последующих этапов проекта с возможным отказом от них.

Высокая доля неуправляемых факторов, преобладание стохастических и неопределенных факторов (Андреев и др., 2002) означают необходимость применения более сложных методик оценки рисков проекта. При этом, как было показано выше, используемые методы оценки риска должны комплексно учитывать экономические, политические, экологические и технологические риски, характерные для магистральных газопроводов.

Учитывая все вышесказанное, для оценки рисков газопроводных проектов представляется правильным применять метод многоуровневого иерархического анализа Саати (Saaty, 1980). Метод заключается в последовательном разделении задачи (в данном случае оценки рисков проекта) на элементарные составляющие ${ }^{17}$, которые затем попарно сравниваются, что упрощает проблему по сравнению с необходимостью единовременной оценки 20-30 рисков различных типов, присущих крупному инфраструктурному проекту. При этом все риски проекта могут быть разделены на группы (экономические, политические, экологические, и т.п.), внутри которых методом Саати проводятся попарные сравнения каждого конкретного риска, а затем та же процедура повторяется для сравнения групп рисков. После проведения экспертной оценки полученные результаты синтезируются для представления рекомендаций об относительной опасности или вероятности реализации рисков проекта. В российской литературе данный метод применительно к оценке рисков газопроводных проектов использовался в работах Порфирьева и других авторов (Порфирьев, 2007а; Порфирьев, 2007б; Белова и др., 2011).

\section{Методика динамической оценки эффективности}

Так как предлагаемую методику предполагается применять для оценки эффективности российских проектов, она должна находиться в соответствии с общими положениями официальных российских методических рекомендаций (Методические рекомендации, 2000). Также в силу необходимости учета нефинансовых составляющих оценки (Lopes, Flavell, 1998), что, как показано в предыдущем разделе, особенно важно для проектов газовой отрасли, в статический блок оценки эффективности подключены элементы комплексной методики оценки, предложенные в работе Дэй (Dey, 2002).

Общий алгоритм предлагаемой методики представлен на рисунке 2. Техническая, экологическая, политическая и общественная (социально-экономическая) оценки позволяют на первом этапе отсеять часть альтернативных вариантов проекта, которые не представляется возможным реализовать, а также ранжировать оставшиеся варианты (с помощью метода Саати), чтобы наилучшие из них перешли на этап оценки коммерческой эффективности проекта в целом. На всех этапах предварительная оценка нефинансовых аспектов дает информацию по ряду предпосылок в оценке коммерческой эффективности и оценке рисков. При получении положительной оценки общественной эффективности проекта должна быть учтена возможность получения государственной поддержки для проекта, которую необходимо включать в проведение оценки коммерческой эффективности (Методические рекомендации, 2000). Оценка общественной эффективности может быть проведена путем расчета мультипликативного эффекта на ВВП от инвестиций в проект по таблицам «Затраты - Выпуск по стране», оценки дополнительной занятости населения и доходов бюджета.

\footnotetext{
17 Например, риск пересмотра заключенных контрактов на поставку газа или риск недостаточного объема инвестиций в добычу газа
} 


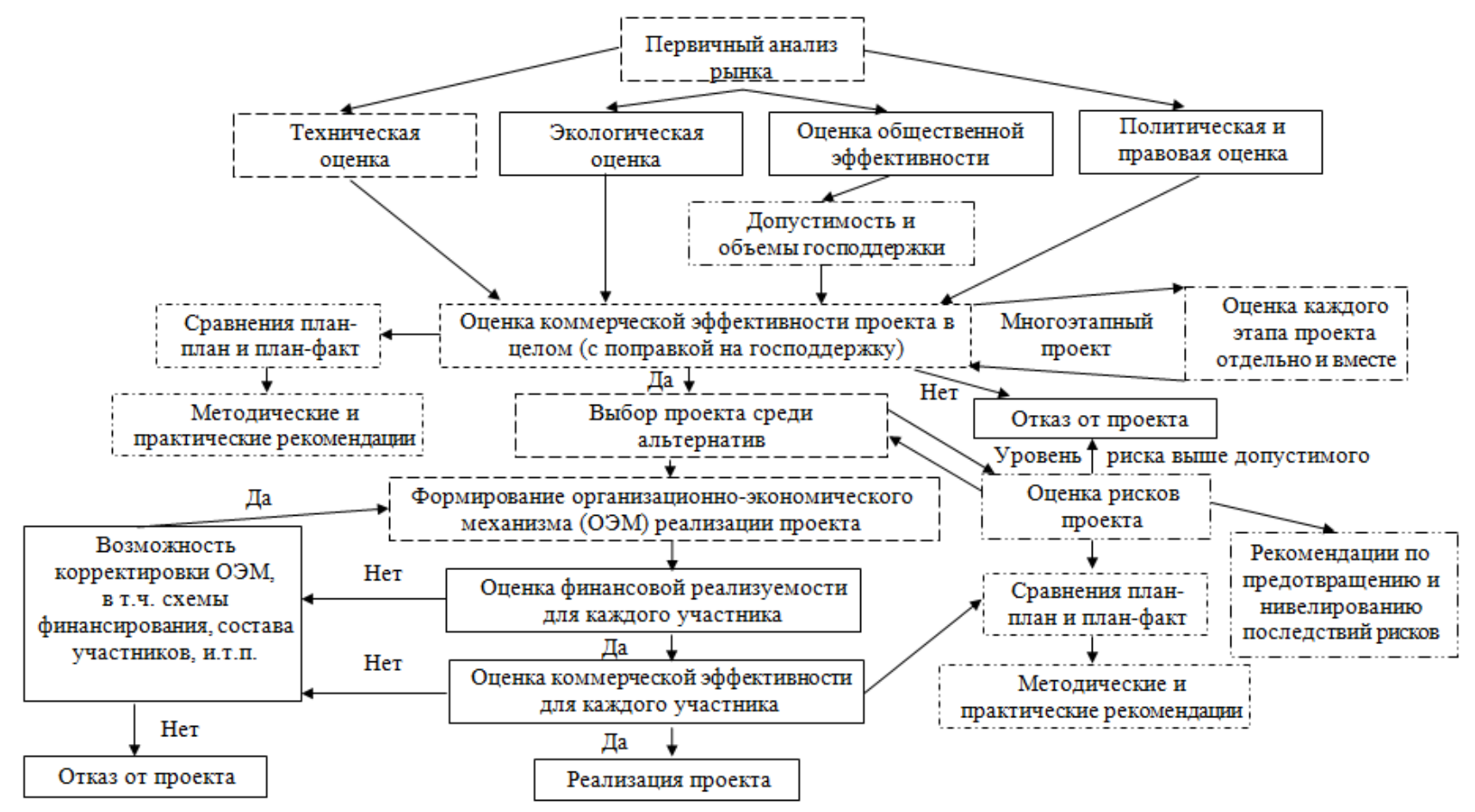

Рисунок 2. Общая схема проведения динамической оценки эффективности инвестиционного проекта

Примечание: Тип линии обозначает, на какой стадии применяется данный шаг оценки. - применяется на всех стадиях, - - только на 1-й стадии, - - - на 1-й и 3-й стадиях, -.- на 2-й и 3-й стадиях, …- на 1-й и 2-й стадиях. Если какой-то из шагов схемы оказывается пропущен на определенной стадии, осуществляется переход к следующему шагу по стрелке.

Следующий этап - оценка коммерческой эффективности проекта в целом, которую, как отмечалось выше, не обязательно проводить на второй стадии. Для оценки коммерческой эффективности может использоваться стандартная методика DCF, но с учетом изложенных в первом разделе особенностей оценки на каждой стадии (в первую очередь относительно вложений прошлых периодов и детальности анализа). В общем случае, учитывая уникальность крупных газопроводных проектов, а также обусловленное большой величиной капиталовложений использование проектного финансирования (а значит, выделения проекта в отдельную частную компанию), в качестве ставки дисконтирования следует использовать средневзвешенную стоимость капитала (WACC). В качестве требуемой доходности на собственный капитал при оценке проекта в целом можно принять средневзвешенную рентабельность инвестированного капитала (ROIC) компаний - участников проекта (веса определятся долями в проекте). При этом модель САРМ оказывается неприменима для подавляющего большинства проектов газопроводов из-за невозможности определить меру систематического риска.

С учетом специфики газовой отрасли при наличии у проекта нескольких этапов (фаз освоения месторождения или веток газопровода) вводится дополнительный шаг: проводится отдельная оценка коммерческой эффективности каждого этапа и их комбинаций. По итогам проведения оценки в целом делается выбор наиболее эффективного варианта проекта (на первой стадии), переход к следующему этапу оценки (вторая и третья стадии) или отказ от проекта при недостаточном уровне прибыльности.

Следующий шаг - оценка рисков - начинается со стандартной проверки чувствительности результирующих показателей коммерческой эффективности (NPV, IRR) не менее чем по пяти параметрам (цена конечной продукции, капитальные вложения, стоимость 
сырья, операционные издержки, курс валют). При этом рентабельность проекта в общем случае должна оставаться положительной при отклонении любого из параметров на $30 \%$.

Оценка рисков проекта может также привести к отказу от него, если уровень риска окажется выше допустимого. В случае применения метода Саати для оценки рисков отказ от проекта может происходить при большей подверженности более опасным рискам выбранной альтернативы по сравнению с другими вариантами проекта.

Таблица 3

Матрица сравнения факторов в методике Саати

\begin{tabular}{|c|c|c|c|c|}
\hline $\begin{array}{c}\text { Параметр (риск, группа } \\
\text { рисков, газопровод) }\end{array}$ & 1 & 2 & $i$ & $n$ \\
\hline 1 & 1 & $a_{12}$ & $a_{1 i}$ & $a_{1 n}$ \\
\hline 2 & $1 / a_{12}$ & 1 & $a_{2 i}$ & $a_{2 n}$ \\
\hline$i$ & $1 / a_{1 i}$ & $1 / a_{2 i}$ & 1 & $a_{i n}$ \\
\hline$n$ & $1 / a_{1 n}$ & $1 / a_{2 n}$ & $1 / a_{i n}$ & 1 \\
\hline
\end{tabular}

Алгоритм проведения оценки рисков методикой Саати выглядит следующим образом. На первом этапе выявляются и описываются группы рисков, присущие подобным проектам (политические, конъюнктурные, финансово-экономические, экологические, технические, ресурсные, институциональные и другие) (Порфирьев, 2007a), а также индивидуальные риски внутри каждой группы, способные иметь влияние на конкретный рассматриваемый проект.

Затем группа экспертов (или один исследователь в упрощенном случае) попарно сравнивает по шкале от 1 до 9 все параметры, как это показано в таблице 3, определяя относительную меру значимости $\left(a_{i j}\right)$ каждого параметра в сравнении с другими. При этом сравнение проводится в трех плоскостях:

1. Для проекта в целом оценивается опасность каждой группы рисков

2. По каждой группе рисков проводится сравнение вариантов проекта (различная мощность, различные маршруты, и.т.п.) по степени подверженности данной группе рисков.

3. Для выбранного варианта проводятся сравнения опасности конкретных рисков внутри каждой группы рисков.

Во всех случаях для полученной матрицы рассчитывается главный собственный вектор $^{18}$, который после нормализации становится вектором приоритетов, отражающим меру значимости каждого фактора относительно других факторов. Зная подверженность вариантов газопроводов каждой группе рисков и их относительную опасность, можно рассчитать предпочтительность вариантов строительства газопровода по величине интегрального риска. При этом выбранный на этапе оценки коммерческой эффективности вариант может быть отклонен, если для этого варианта уровень интегрального риска оказывается существенно выше, чем для альтернатив (при этом нельзя предложить однозначное решение, при каких значениях проект должен отклоняться, - это в значительной степени зависит от принятой в компании политики управления рисками).

На основе оценки относительной значимости групп рисков и конкретных рисков внутри каждой группы можно выделить наиболее опасные риски. Для предотвращения или нивелирования последствий этих рисков компании-оператору проекта следует запланировать соответствующие мероприятия. Например, если в число значимых попал экологический риск нанесения ущерба популяции рыб в акватории, можно скорректировать маршруты, материалы и период строительства газопровода для его предотвращения. Если в качестве

\footnotetext{
18 Перемножением $n$ элементов каждой строки и извлечением корня степени $n$ при проведении примерной оценки или возведением матрицы в произвольно большие степени с последующим делением суммы каждой строки на общую сумму элементов матрицы при точной оценке (Saaty, 1980).
} 
значимого определен риск временных перебоев в поставках газа, можно предусмотреть в проекте строительство подземного хранилища газа.

По итогам оценки рисков методом Саати в анализ коммерческой эффективности проекта в дополнение к рассчитанному базовому сценарию добавляются альтернативные сценарии, основанные на наиболее вероятных и опасных рисках. При этом сценарии должны основываться на связанном наборе допущений. В целом для российских экспортных газопроводов наибольшее влияние оказывают политические риски (Белова и др., 2011), поэтому стандартным альтернативным сценарием для них является противодействие властей на рынках сбыта. В частности, для европейских газопроводов такой сценарий может включать в себя одновременно:

- диверсификацию импорта газа и снижение его доли в энергобалансе (приводит к уменьшению объема поставок и снижению цен);

- задержку в получении разрешений на строительство (отсрочка проекта на срок от полугода и более);

- введение законодательных норм по разграничению деятельности компаний по видам деятельности (отказ от поставок газа конечным потребителям по более высоким ценам);

- отказ от выдачи льготных кредитов экспортно-импортных агентств (увеличение процентных расходов);

• повышенное внимание регуляторов (дополнительные расходы на экологические и PRмероприятия).

В каждом из альтернативных сценариев проект должен оставаться рентабельным.

Если наиболее выгодный проект отклонен из-за слишком высокого уровня риска, то проводится оценка риска следующего проекта по степени привлекательности при условии достаточного уровня эффективности до тех пор, пока не будет выбран устраивающий вариант или не произойдет отклонение проекта. Если риск признается допустимым, то итогом этого блока оценки становятся рекомендации по снижению вероятности возникновения рисков и уменьшению ущерба, если они все же исполнились.

Следующий важный шаг после оценки проекта в целом и оценки рисков - его оценка для каждого из участников. Для этого в первую очередь формируется организационноэкономический механизм проекта (ОЭМ), который затем может модифицироваться, если не достигается финансовая реализуемость и рентабельность для каждого участника (Методические рекомендации, 2000). Следует учитывать, что набор выгод и издержек участника проекта может существенно отличаться от других участников и проекта в целом. Это зависит как от ОЭМ (например, объема вложений и получаемых выгод участника), особенностей бизнеса участника (например, для «Газпрома» основные выгоды связаны с продажей газа крупным европейским компаниям, для прочих участников - с перепродажей газа конечным потребителям и платой за транспортировку). В качестве дисконт-фактора может применяться ROIC каждого из участников. При положительном прохождении этого этапа оценки выдается окончательная рекомендация об эффективности реализации проекта.

После этапов оценки коммерческой эффективности в целом и для участников проекта, а также оценки рисков на второй и третьей стадиях проекта должны проводиться сравнения план-план и план-факт. Итогом этого блока должны быть рекомендации по изменению или замене используемых методик, предпосылкам при проведении будущих оценок, потенциальной успешности будущих аналогичных проектов. Следует учитывать, что даже если переоценка рисков на третьей стадии не проводится, то все равно необходимо сравнить проведенные ранее оценки с фактически случившимися рисками и величинами ущерба от них.

С помощью блока сравнений план-план и план-факт можно рассчитать коэффициенты отклонения, характерные для проектов определенного типа (например, магистральных газопроводов), как это сделано в работе Пола и Михалека (Pohle, Mihaljek, 1992). Затем полученные коэффициенты можно использовать в качестве поправочных при проведении оценки эффективности новых проектов, таких как «Южный Поток», «Алтай, Дальний 
Восток - Южная Корея», «Восточная Сибирь - Китай» и других.

\section{Заключение}

В работе представлена методика динамической оценки эффективности инвестиционных проектов, которая предполагает модификацию используемых инструментов оценки для каждой из трех стадий жизненного цикла проекта: подготовки, реализации и эксплуатации. Первопричиной различий оценки на трех стадиях является основная цель ее проведения: принятие решения об инвестировании в конкретный вариант проекта на стадии подготовки, мониторинг состояния и рисков проекта при реализации и завершающая оценка во время эксплуатации проекта. Дается подробное описание расхождений в методиках проведения оценки на каждой стадии, которые могут заключаться в наличии или отсутствии какого-то шага (сравнений план-факт, оценки проекта в целом, выборов проекта среди альтернатив), уровнем доступной информации и неопределенности, влияющих на выбор конкретных методик и инструментов оценки.

Методика построена с учетом особенностей газовых проектов в целом и газопроводных в частности. Для этого разработана матрица особенностей проекта, построенная в двух осях: неотъемлемые - распространенные и отраслевые - трубопроводные особенности. На основе анализа особенностей выявлено, что проведение динамической оценки эффективности является самой актуальной задачей именно для газовых проектов, указана важность учета нефинансовых аспектов оценки (в первую очередь политических и экологических факторов). Предложен оптимальный подход к оценке рисков газопроводных проектов - метод Саати.

На основании проведенного анализа представлена общая методика оценки проектов, не противоречащая российским методическим рекомендациям и в то же время использующая зарубежные наработки по оценке и учитывающая особенности газовых проектов (нефинансовые аспекты и многоэтапность проектов). Методика модифицирована с учетом особенностей проведения оценки на каждой стадии проекта, что позволяет проводить с ее помощью динамическую оценку эффективности.

Внедрение подобной методики позволит, с одной стороны, оптимизировать процесс проведения оценок, а с другой - дать практические и методические рекомендации при оценке новых инвестиционных проектов, что может привести к отсеву большего числа неэффективных проектов (особенно в газовой отрасли) и тем самым будет способствовать увеличению эффективности российских компаний и экономики в целом.

\section{Список литературы}

1. Андреев А.Ф. Оценка эффективности и планирование проектных решений в нефтегазовой промышленности. М.: Нефть и газ, 1997.

2. Андреев А.Ф., Зубарева В.Д., Курпитко В.Г., Саркисов А.С. Оценка рисков нефтегазовых проектов. М.: Нефть и газ, 2002.

3. Башмаков И. Будет ли экономический рост в России в середине XXI века? // Вопросы экономики. 2011. № 3. С. 20-39.

4. Белова М., Минуллин Я., Порфирьев Б., Протасов В., Фейгин В. Комплексная оценка рисков физической безопасности нефте- и газоэкспортной инфраструктуры России // Таможенное обозрение. 2011. № 1. С. 45-51.

5. Виленский П.Л., Лившиц В.Н., Смоляк С.А. Оценка эффективности инвестиционных проектов. Теория и практика. М.: Дело, 2008.

6. Волков И.М., Грачева М.В., Проектный анализ. М.: Инфра-М, 2009.

7. Всемирный экономический форум, Евразийский институт конкурентоспособности. Доклад о конкурентоспособности России 2011: закладывая фундамент устойчивого процветания / под. ред. А. Праздничных, M. Хануз. 2011. URL: http://www.weforum.org/reports/russia-competitiveness-report-2011. 
8. Зубарева В.Д. Проблемы комплексной экономической оценки проектных решений в нефтегазовой промышленности: дисс... на соискание ученой степени д-ра эконом. наук. М., 2001.

9. Игнатьев М. Трубный рынок: могло быть хуже // Нефтегазовая вертикаль. 2010. № 7. C. $46-51$.

10. Клейнер В., Корпоративное управление и эффективность деятельности компании (на примере ОАО «Газпром») // Вопросы экономики. 2006. № 3. С. 86-103.

11. Лившиц В.Н., Швецов А.Н. Каких ошибок следует избегать при оценке инвестиционных проектов с участием государства // Вопросы экономики. 2011. № 9. С. $80-92$.

12. Методические рекомендации по оценке эффективности инвестиционных проектов.. М.: Экономика, 2000.

13. Милов В. Энергодиалог Россия - EC: заполнить вакуум // Россия в глобальной политике. 2007. № 5. С. 135-146.

14. Миловидов К.Н., Коржубаев А.Г., Эдер Л.В. Нефтегазообеспечение глобальной экономики. М.: ЦентрЛитНефтеГаз, 2006.

15. Порфирьев Б.Н. Сравнительная оценка рисков транспортировки газа с острова Сахалин на Дальний Восток и в Республику Корея // Пространственная экономика. 2007a. № 3. С. 5-20.

16. Порфирьев Б.Н. Оценка рисков проекта газопровода из России в Китай // ЭКО. Всероссийский экономический журнал. 2007б. № 11. С. 79-93.

17. Энергетическая стратегия России на период до 2030 г. Утверждена распоряжением Правительства Российской Федерации от 13 ноября 2009 г. № 1715-p. URL http://www.energystrategy.ru/projects/docs/ES-2030_(utv._N1715-p_13.11.09).doc.

18. Anbari, F.T., Carayannis, E.G., and Voetsch, R.G. (2008), Post-project reviews as a key project management competence, Technovation, 28 (2008) 633-643.

19. Anbari, F.T. (1985), A systems approach to project evaluation, Project Management Journal, 16 (1985) 21-26.

20. Baev, P., Overland, I., (2010), International Affairs, 5(86) (2010) 1075-1090.

21. Belli, P., Anderson, J., Barnum, H., Dixon, J., and Tan J-P. (1998), Handbook on economic analysis of investment operations, Operational Core Services Network Learning and Leadership Center.

22. Busby, J.S. (1999), An assessment of post-project reviews, Project Management Journal, 30 (1999) 23-29.

23. Cano, A. (1992), Continuous project feasibility study and continuous project risk assessment, International Journal of Project Management, 3(10) (1992) 165-170.

24. Cleland, D.I. (1985), A strategy for ongoing project evaluation, Project Management Journal, 16 (1985) 11-17.

25. Dey, P.K. (2002), An integrated assessment model for cross-country pipelines, Environmental Impact Assessment Review, 22 (2002) 703-721.

26. Kaufman, D. (1991), The forgotten rationale for policy reform: the productivity of investment, background study for the World Bank's World Development Report, World Bank, Washington.

27. Erikstad, L., Lindblom, I., Jerpasen, G., Hanssen, M., Bekkby, T., Stabbetorp, O., and Bakkestuen, V. (2008), Environmental value assessment in a multidisciplinary EIA setting, Environmental Impact Assessment Review, 2-3(28) (2008) 131-143.

28. Gunton, T. (2003), Megaprojects and Regional Development: Pathologies in Project Planning, Regional Studies, 5(37) (2003) 505-519.

29. Huseby, A., and Skogen, S. (1992), Dynamic risk analysis: the DynRisk concept International Journal of Project Management, 3(10) (1992) 160-164.

30. International Energy Agency (2010), World Energy Outlook 2010. IEA Publications, Paris.

31. Labuschagne, C., and Brent, A.C. (2005), International Journal of Project Management, 23 
(2005) 159-168.

32. Larsson, R. (2007), Nord Stream, Sweden and Baltic Sea Security, FOI papers.

33. Lester, A. (2007), Project Management, Planning and Control (Fifth Edition). Oxford.: Butterworth-Heineman.

34. Lopes, M.D., Flavell, R. (1998), International Journal of Project Management, 4(16) (1998) 223-233.

35. Olsson, N., Krane, H.P., Rolstadas, A., and Veiseth, M. (2010), Transport Policy, 17 (2010) 251-258.

36. Pohle, G., and Mihaljek, D., (1992), Project Evaluation and Uncertainty in Practice: A Statistical Analysis of Rate-of-Return Divergences of 1,015 World Bank Projects, The World Bank Economic Review, 2(6) (1992) 255-277.

37. Saaty, T.L. (1980), The analytic hierarchy process. New York: McGraw-Hill.

38. Xie, G., Yue, W., Wang, S., and Lai, K. (2010), Dynamic risk management in petroleum project investment based on a variable precision rough set model, Technological Forecasting \& Social Change, 77 (2010) 891-901. 